\title{
Optimal Selection of Gear Ratio for Hybrid Electric Vehicles Using Modern Meta-Heuristics Search Algorithm
}

\author{
Vikram Kumar Kamboj ${ }^{1}$, Sobhit Saxena ${ }^{2}$, Kamalpreet Sandhu ${ }^{3}$ \\ ${ }^{1,2}$ School of Electronics and Electrical Engineering, Lovely Professional University, Punjab, INDIA \\ ${ }^{3}$ Product and Industrial Design Department, School of Design, Lovely Professional University, Phagwara, INDIA
}

\begin{abstract}
Gear Train Design problem is most important design problem for machine tools manufacturers. Recent work on gear train improvement has been bound towards multi-shaft gear trains of the speed-change kind, where major focus is to maximize the range of operating speeds and to minimize the number of gears and spindles. In the proposed research, a hybrid meta-heuristic search algorithm is presented to design and optimize multi-spindle gear trains problem. The objective of the research is to optimize gear trains on the basis of minimum overall centre distance, minimum overall size, minimum gear volume, or other desirable criteria, such as maximum contact or overlap ratios. The proposed hybrid meta-heuristic search algorithm is inspired by canis lupus family of grey wolves and exploitation capability of existing grey wolf optimizer is further enhanced by pattern search algorithm, which is a derivative-free, direct search optimization algorithm suitable for non-differential, discontinuous search space and does not require gradient for numerical optimization problem and have good exploitation capability in local search space. The effectiveness of the proposed algorithm has been tested on various mechanical and civil design problem including gear train design problem, which includes four different gear and experimental results are compared with others recently reported heuristics and meta-heuristics search algorithm. It has been found that the proposed algorithm indorses its effectiveness in the field of nature inspired meta heuristics algorithms for engineering design problems for hybrid electric vehicles.
\end{abstract}

Keywords. Engineering Design Problems, Gear Train Design Problem, Hybrid Electric Vehicles, Meta-Heuristics. 


\section{INTRODUCTION}

Multidisciplinary design optimization and multidisciplinary system design optimization are emerging area for the solution of design and optimization problems incorporating a number of disciplines. With the advancement in technology a new era of problem solving methods is emerging which make use of computers. Optimization techniques are considered to be one of the best tool for solving the engineering problems and to find the optimal results for the problem. These approaches consider the problem as black box and find the optimal solution. The optimization process initialize with random set for specified problem and then improving them over predefined steps. The engineering problems to be tackled consist of various difficulties such as constraints, uncertainties, local solution, multiple objective, etc. Optimization technique should be able to discourse these issues. While evaluating the optimization problem exploration and exploitation are the criteria to be taken into account based on these two features the algorithms are classified into two categories consisting of population based search algorithm which is exploration oriented and the other one is evolution based algorithms which are exploitation focused and there should be a good balance between them so as to enhance the working efficiency of the resultant algorithm. One of the method to achieve this balance is by using hybrid algorithm which enhance performance by combining two techniques the resulting technique is called memetic algorithm. In the recent years, various meta-heuristics search algorithms has been implemented such as Biogeography based Optimizer [1], Grey Wolf Optimizer [2], Ant Lion Optimizer [3], Moth Flame Optimizer [4], Multi Verse Optimizer [5], Dragon Fly Algorithm [6],Sine Cosine Algorithm [7],Lightning Search Algorithm [8], Seeker Optimization Algorithm [9],Virus Colony Search Algorithm [10], Whale Optimization Algorithm [11], Wind Driven Optimization [12]. However, some certifiable designing and logical improvement issues are exceptionally intricate and hard to settle, utilizing these techniques. On the off chance that there is more than one neighborhood minima in the problem, the outcome may rely upon the choice of an underlying point, and the acquired minima may not really be the worldwide minima. Moreover, the gradient search may end up plainly troublesome and unstable when the target work and, imperatives have different or sharp peaks. The computational disadvantages of existing numerical straight and nonlinear strategies have constrained analysts to depend on meta-heuristic calculations in light of reproductions to take care of building and logical streamlining issues. A few traditional techniques are accessible to take care of the unit commitment issue. Be that as it may, every one of these strategies require the correct numerical model of the framework and there is a shot of stalling out at the nearby optima. Also, The No-Free-Lunch theorem for optimization allow developers to develop new algorithm or to improve the existing algorithm because, it logically proves that there is no such optimization algorithm which can solve all the optimization problems with equal efficiency for all. Some algorithm work best for few problems and worst for the rest of the problems. So, there is always a scope or improvement to develop the algorithm which could work well for most of the problems.

\section{PATTERN SERACH AGORITHM}

Pattern search method, also known as black box method, is a derivative free method having local search capability and suitable for search problem, where the derivative of the objective function is inconvenient or unknown. The method involve two moves while performing its operation: one is exploratory search which is local search looking for improving the direction to be moved the other move is the pattern move which is a larger search for improving the direction in this move step size is increased unless the improvement is not altered.

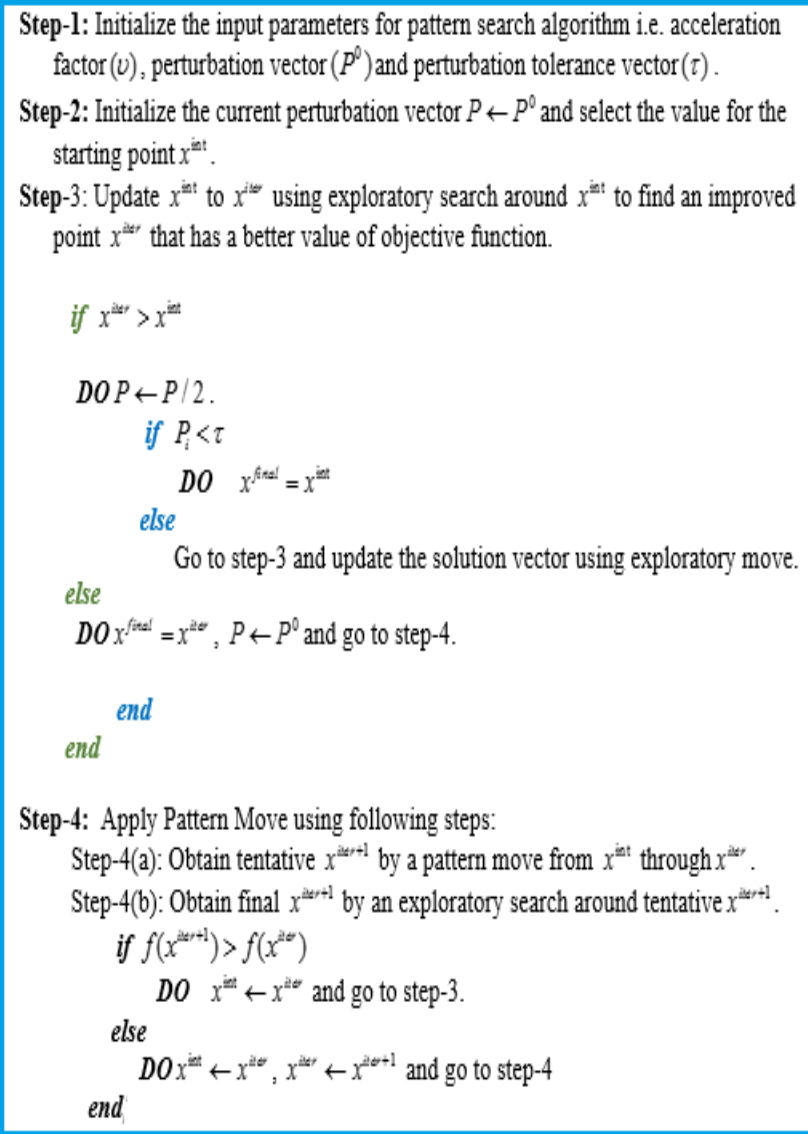

Fig.1. The PSEUDO code for the Pattern Search algorithm

The pattern move require two points one is the current point and the other one is some random point having better value of the objective function which guides the search direction the consideration of new point is guided by equation (1) $\quad x^{\text {iter }+1}=x^{\text {int }}+v\left[x^{\text {iter }}-x^{\text {int }}\right]$ 
(1) Where, $v$ is positive acceleration factor, which is used to multiply the length of the direction improvement vector. The PSEUDO code for the Pattern Search algorithm is shown in Fig.1.

\section{HYBRID GREY WOLF OPTIMIZER}

Grey Wolf Optimizer [13] is swarm intelligence-based, recently developed, metaheuristics search algorithm inspired from the hunting mechanism and leadership hierarchy of grey wolves in nature and require few control parameters and was initially applied to solve 29 benchmark problems and three classical engineering design problems such as tension/compression spring, welded beam, pressure vessel designs problem and real world optical engineering. Further, it was successfully applied to solve various Engineering Optimization Problems such as Economic Load Dispatch Problem [14], Economic load dispatch problem with valve point effect [15], Unit Commitment Problem and training multi-layer perceptron [17]. Several algorithms have also been developed to improve the convergence performance of Grey Wolf Optimizer that includes parallelized GWO [18], hybrid GWO with Genetic Algorithm (GA) [19], Hybrid DE with GWO [20] , Hybrid Grey Wolf Optimizer using Elite Opposition Based Learning Strategy and Simplex Method [21], Modified Grey Wolf Optimizer (mGWO) [22], Mean Grey Wolf Optimizer (MGWO) [23], Hybrid Particle Swarm Optimization with Grey Wolf Optimizer (HPSOGWO) [24] and RWGWO [25]. In the proposed research, the authors has further tried to improve the exploration phase of the existing grey wolf optimizer algorithm

\subsection{PROBLEM FORMULATION}

Primarily developed Grey Wolf Optimizer, is a transformative calculation algorithm, based on grey wolves, which recreate the social level and hunting component of grey wolves in view of three principle ventures of chasing: scanning for prey, encompassing prey and assaulting prey and its mathematically model was designed in view point of hierarchy level of different wolves. The fittest solution was designated as alpha $(\alpha)$. Accordingly, the second and third best solutions are named beta $(\beta)$ and delta $(\delta)$ individually. Whatever is left of the hopeful solution are thought to be kappa $(\kappa)$, lambda $(\lambda)$ and omega $(\omega)$. For the fitness value calculation, the advancement (i.e. chasing) is guided by $\alpha, \beta$ and $\delta$. The $\omega, \kappa$ and $\lambda$ wolves trail these three wolves. In GWO, Encircling or Trapping of Prey was achieved by calculating $\overrightarrow{\mathrm{D}}$ and $\overrightarrow{\mathrm{X}}_{\mathrm{GWolf}}$ vectors described by equations (2) and (3).

$$
\begin{aligned}
& \overrightarrow{\mathrm{D}}=\mid \overrightarrow{\mathrm{C}} \cdot \overrightarrow{\mathrm{X}}_{\text {Prey }}(\text { iter })-\overrightarrow{\mathrm{X}}_{\text {GWolf }} \text { (iter) } \mid \\
& \overrightarrow{\mathrm{X}}_{\text {GWolf }}(\text { iter }+1)=\overrightarrow{\mathrm{X}}_{\text {Prey }}(\text { iter })-\overrightarrow{\mathrm{A}} \cdot \overrightarrow{\mathrm{D}}
\end{aligned}
$$

Where, iter demonstrates the present iteration, $\vec{A}$ and $\vec{C}$ are coefficient vectors, $\overrightarrow{\mathrm{X}}_{\text {Prey }}$ is the position vector of the prey and $\vec{X}_{\text {GWolf }}$ shows the position vector of a grey wolf and the vectors $\overrightarrow{\mathrm{A}}$ and $\overrightarrow{\mathrm{C}}$ are calculated as follows:

$$
\overrightarrow{\mathrm{A}}=2 \overrightarrow{\mathrm{a}} \cdot \vec{\mu}_{1}-\overrightarrow{\mathrm{a}}
$$$$
\overrightarrow{\mathrm{C}}=2 \cdot \vec{\mu}_{2}
$$

Where, $\vec{\mu}_{1}, \vec{\mu}_{2} \in \operatorname{rand}(0,1)$ and $\vec{a}$ decreases linearly from 2 to 0 . The hunting of prey are achieved by calculating the corresponding fitness score and positions of alpha, beta and delta wolves using equations (6), (7) and (8) respectively and final position for attacking towards the prey was calculated by equation (9).

$$
\begin{aligned}
& \overrightarrow{\mathrm{D}}_{\text {Alpha }}=\operatorname{abs}\left(\overrightarrow{\mathrm{C}}_{1} \cdot \overrightarrow{\mathrm{X}}_{\text {Alpha }}-\overrightarrow{\mathrm{X}}\right) \\
& \overrightarrow{\mathrm{X}}_{1}=\overrightarrow{\mathrm{X}}_{\text {Alpha }}-\overrightarrow{\mathrm{A}}_{1} \cdot \overrightarrow{\mathrm{D}}_{\text {Alpha }} \\
& \overrightarrow{\mathrm{D}}_{\text {Beta }}=\operatorname{abs}\left(\overrightarrow{\mathrm{C}}_{2} \cdot \overrightarrow{\mathrm{X}}_{\text {Beta }}-\overrightarrow{\mathrm{X}}\right) \\
& \overrightarrow{\mathrm{X}}_{2}=\overrightarrow{\mathrm{X}}_{\text {Beta }}-\overrightarrow{\mathrm{A}}_{2} \cdot \overrightarrow{\mathrm{D}}_{\text {Beta }} \\
& \overrightarrow{\mathrm{D}}_{\text {Delta }}=\operatorname{abs}\left(\overrightarrow{\mathrm{C}}_{3} \cdot \overrightarrow{\mathrm{X}}_{\text {Delta }}-\overrightarrow{\mathrm{X}}\right) \\
& \overrightarrow{\mathrm{X}}_{3}=\overrightarrow{\mathrm{X}}_{\text {Delta }}-\overrightarrow{\mathrm{A}}_{3} \cdot \overrightarrow{\mathrm{D}}_{\text {Delta }} \\
& \overrightarrow{\mathrm{X}}(\text { iter }+1)=\frac{\left(\overrightarrow{\mathrm{X}}_{1}+\overrightarrow{\mathrm{X}}_{2}+\overrightarrow{\mathrm{X}}_{3}\right)}{3}
\end{aligned}
$$

In the proposed hybrid Grey-Wolf Optimizer-Pattern Search (hGWO-PS) algorithm, the randomly generated position vector $\overrightarrow{\mathrm{X}}$ has been further modified using Pattern search method and the modified position vector $\overrightarrow{\mathrm{X}}$, has been applied to grey wolves to evaluate alpha, beta and delta scores. In order to hybridize the GWO and PS, the heuristics procedure has been adopted. The exploration phase in hGWO-PS is similar to classical GWO. In order to explore the search space globally, vector $\vec{A}$ and $\vec{C}$ are used, which mathematically model divergence. The absolute value of $\vec{A}$ greater than 1 forces the grey wolves to diverge from the prey to optimistically find an adequate prey. The PSEUDO code of proposed hGWO-PS algorithm has been shown in Fig.2. 


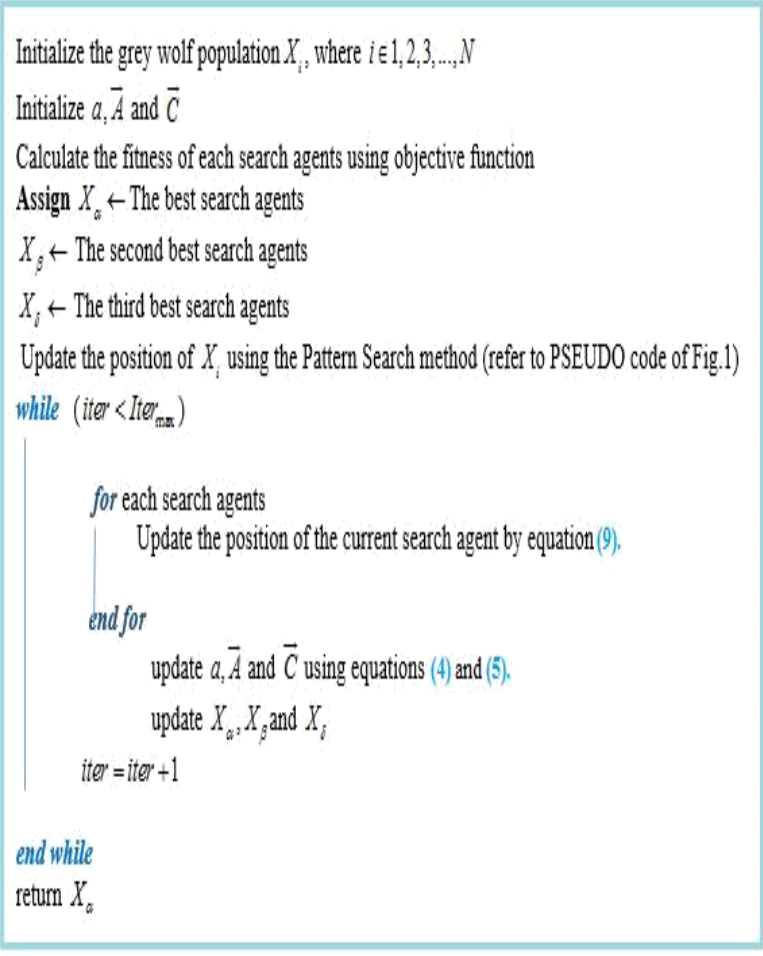

Fig 2: PSEUDO code of proposed hGWO-PS algorithm

\section{ENGINEERING OPTIMIZATION PROBLEMS}

In order to validate the performance of the proposed hGWO-PS algorithm, six engineering design optimization problems are taken into consideration which includes Three-bar truss problem, Pressure vessel problem, Tension/compression spring design problem, welded beam problem, Cantilever Beam Design problem and Gear Train design problem (Fig.3 to Fig.8). In order to validate the stochastic nature of proposed algorithm, 30 trial runs has been performed and results are evaluated for mean, worst and best values of fitness including standard deviation. In the whole research study, 30 search agents are taken into considerations and algorithm is simulated for maximum iterations of 1500 . For the effective analysis of results, the non-parametric statistical hypothesis test i.e. Wilcoxon rank sum test has been applied. The significance of the test is to examine the distribution of samples i.e. whether two dependent samples selected from populations having the same distribution or not. The results of the engineering optimization design problems are recorded with respect to best value, worst value, mean value, standard deviation and p-value and has been reported in Table-1. The comparison of results for hGWO-PS has been shown in Table-1 through Table-2. The results of aforementioned engineering problems strongly evidence the merits of the hGWO-PS algorithm in solving problems with unknown search spaces. The results also show that the proposed algorithm is suitable for constrained and discrete problems. The convergence curve and trial runs solutions for these engineering problems has been depicted in Fig.8 and Fig.9 respectively, which shows the superiority of hGWO-PS over GWO.

\subsection{Three-Bar Truss Design Problem}

In order to validate the results of proposed hGWO-PS algorithm for Engineering optimization problems, the first problem of a three-bar truss design has be undertaken, where the objective of the fitness function is to minimize its weight. The various constrains for threebar truss design problem are stress constraint, deflection constraint, and buckling constraint. The mathematical model for the aforementioned problem has been shown below in equations (10) through (12).

Consider $\vec{x}=\left[x_{1}, x_{2}\right]=\left[A_{1}, A_{2}\right]$

Minimize $f(\vec{x})=\left(2 \sqrt{2} x_{1}+x_{2}\right) * l$

Subject to $g_{1}(\vec{x})=\frac{\sqrt{2} x_{1}+x_{2}}{\sqrt{2} x_{1}^{2}+2 x_{1} x_{2}} P-\sigma \leq 0$

$g_{2}(\vec{x})=\frac{x_{2}}{\sqrt{2} x_{1}^{2}+2 x_{1} x_{2}} P-\sigma \leq 0$

$g_{3}(\vec{x})=\frac{1}{\sqrt{2} x_{2}+x_{1}} P-\sigma \leq 0$

Variable range $0 \leq x_{1}, \quad x_{2} \leq 1$

Where $1=100 \mathrm{~cm}, \mathrm{P}=2 \mathrm{KN} / \mathrm{cm}^{2}, \sigma=2 \mathrm{KN} / \mathrm{cm}^{2}$

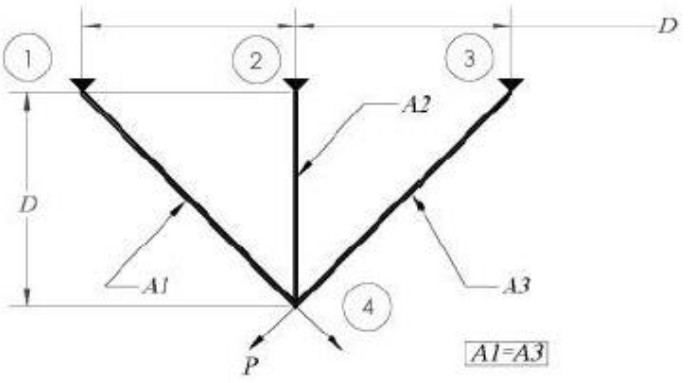

Fig 3: Three-Bar Truss Design Problem

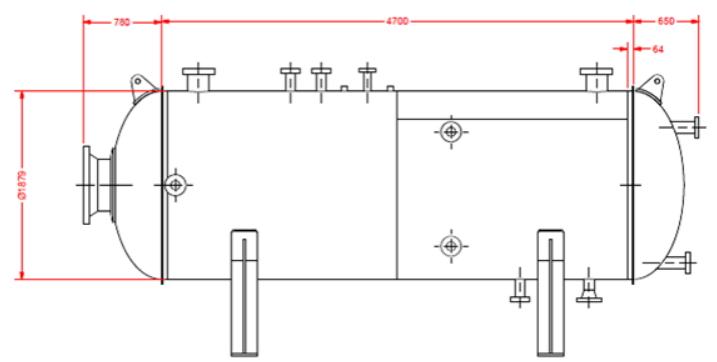

Fig 4: Pressure Vessel Design Problem

\subsection{Pressure Vessel Design Problem}

The second multidisciplinary design optimization problem, which has been taken into consideration is Pressure Vessel Design Problem. The objective function of this problem is to minimize the total cost, which is the cost of material, forming, and welding of a cylindrical vessel (Fig.4). For the designing of pressure vessel problem, four different variables are taken into 
consideration, which are Thickness of the shell (Ts), Thickness of the head (Th), Inner radius (R) and Length of the cylindrical section without considering the head (L). The head of the vessel has been taken in the hemi spherical shape and both ends of the vessel has been capped. The aforementioned design problem is subjected to four constraints. The mathematical formulation of the problem and its constrained is shown below in equations (13) through (14).

Consider $\vec{x}=\left[x_{1} x_{2} x_{3} x_{4}\right]=\left[T_{s} T_{h} R L\right]$

Minimize

$$
\begin{aligned}
& f(\vec{x})=0.6224 x_{1} x_{3} x_{4}+1.7781 x_{2} x_{3}^{2}+3.1661 x_{1}^{2} x_{4} \\
& +19.84 x_{1}^{2} x_{3}
\end{aligned}
$$

Subjected to $\quad g_{1}(\vec{x})=-x_{1}+0.0193 x_{3} \leq 0$ (14a)

$$
\begin{aligned}
& g_{2}(\vec{x})=x_{3}+0.00954 x_{3} \leq 0 \\
& g_{3}(\vec{x})=-\pi x_{3}^{2} x_{4}-\frac{4}{3} \pi x_{3}^{3}+1296000 \leq 0
\end{aligned}
$$

$(14 c)$

$$
g_{4}(\vec{x})=x_{4}-240 \leq 0
$$

Variable range $0 \leq x_{1} \leq 99$

$0 \leq x_{2} \leq 99,10 \leq x_{3} \leq 200,10 \leq x_{4} \leq 200$

\subsection{The Tension/Compression Spring Design Problem}

The third multidisciplinary design optimization, which is taken into consideration for engineering optimization is Tension/Compression Spring Design Problem (Fig.5), which is a kind of mechanical engineering problem. The objective of the spring design problem is to minimize the weight of the spring. To solve the problem, three design variables i.e. the number of active coils $(\mathrm{N})$, mean coil diameter (D) and wire diameter (d) are taken into contemplation and the problem is subjected to surge frequency constraints, shear stress constraints and minimum deflection constraints. The mathematical model of the aforesaid problem is explained below in equations (15) through (16).

Consider $\vec{x}=\left[x_{1} x_{2} x_{3}\right]=[d D N]$,

Minimize $f(\vec{x})=\left(x_{3}+2\right) x_{2} x_{1}^{2}$,

\[ g_{1}(\vec{x})=1-\frac{x_{2}^{3} x_{3}}{71785 x_{1}^{4}} \leq 0 \]
Subjected to

$$
g_{2}(\vec{x})=\frac{4 x_{2}^{2}-x_{1} x_{2}}{12566\left(x_{2} x_{1}^{3}-x_{1}^{4}\right)}+\frac{1}{5108 x_{1}^{2}} \leq 0
$$

$$
\begin{aligned}
& g_{2}(\vec{x})=\frac{4 x_{2}^{2}-x_{1} x_{2}}{12566\left(x_{2} x_{1}^{3}-x_{1}^{4}\right)}+\frac{1}{5108 x_{1}^{2}} \leq 0 \\
& g_{3}(\vec{x})=1-\frac{140.45 x_{1}}{x_{2}^{2} x_{3}} \leq 0 \\
& g_{4}(\vec{x})=\frac{x_{1}+x_{2}}{1.5}-1 \leq 0
\end{aligned}
$$

Variable range $0.005 \leq x_{1} \leq 2.00$

$$
\begin{aligned}
& 0.25 \leq x_{2} \leq 1.30 \\
& 2.00 \leq x_{3} \leq 15.0
\end{aligned}
$$

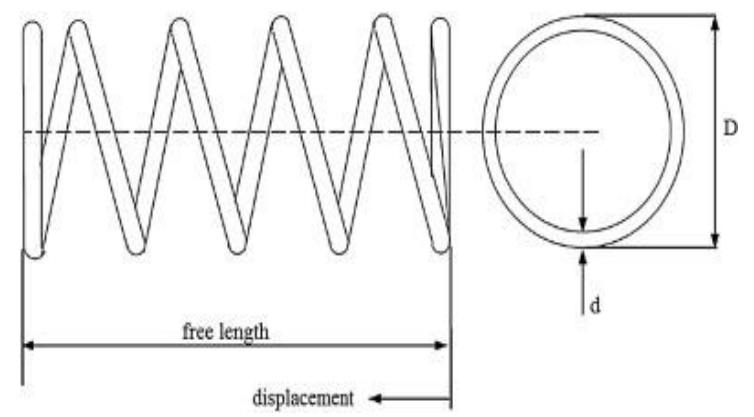

Fig 5: The Tension/Compression Spring Design Problem

\subsection{Welded Beam Design Problem}

The fourth engineering optimization problem, which is taken into consideration is welded beam design problem (Fig.6), where the objective of the problem is to minimize the fabrication cost of a welded beam. The problem consists of four variables, which are length of attached part of bar (1), thickness of the bar (b), the height of the bar (t) and thickness of weld (h). The problem is subjected to four constraints, which includes Side constraint, Buckling load on the bar (Pc), End deflection of the beam (d), Bending stress in the beam (h) and Shear stress (s). The mathematical formulation of the abovementioned problem is explained below in equations (17) through (19).

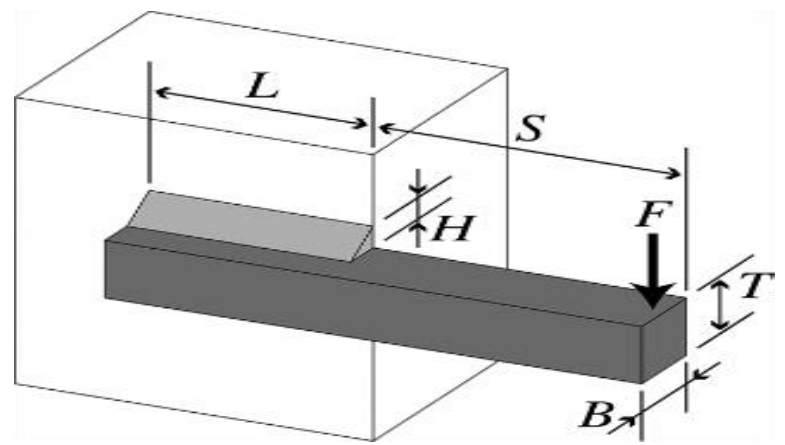

Fig 6: Welded Beam Design Problem

Consider $\vec{x}=\left[x_{1} x_{2} x_{3} x_{4}\right]=[h l t b]$,

Minimize 
$f(\vec{x})=1.10471 x_{1}^{2} x_{2}+0.04811 x_{3} x_{4}\left(14.0+x_{2}\right)$

Subject to $g_{1}(\vec{x})=\tau(\vec{x})-\tau_{\max } \leq 0$,

$g_{2}(\vec{x})=\sigma(\vec{x})-\sigma_{\max } \leq 0$

$g_{3}(\vec{x})=\delta(\vec{x})-\delta_{\max } \leq 0$

$g_{4}(\vec{x})=x_{1}-x_{4} \leq 0$

$g_{5}(\vec{x})=P-P_{c}(\vec{x}) \leq 0$

$g_{6}(\vec{x})=0.125-x_{1} \leq 0$,

$g_{7}(\vec{x})=1.10471 x_{1}^{2}+0.04811 x_{3} x_{4}\left(14.0+x_{2}\right)-5.0 \leq 0$

Variable range $0.1 \leq x_{1} \leq 2,0.1 \leq x_{2} \leq 10$

$0.1 \leq x_{3} \leq 10,0.1 \leq x_{4} \leq 2$

Where $\tau(\vec{x})=\sqrt{\left(\tau^{\prime}\right)^{2}+2 \tau^{\prime} \tau^{\prime \prime} \frac{x_{2}}{2 R}+\left(\tau^{\prime \prime}\right)^{2}}$

$\tau^{\prime}=\frac{P}{\sqrt{2} x_{1} x_{2}}, \tau^{\prime \prime}=\frac{M R}{J}, M=P\left(L+\frac{x_{2}}{2}\right)$,

$R=\sqrt{\frac{x_{2}^{2}}{4}+\left(\frac{x_{1}+x_{3}}{2}\right)^{2}}$,

$J=2\left\{\sqrt{2} x_{1} x_{2}\left[\frac{x_{2}^{2}}{4}+\left(\frac{x_{1}+x_{3}}{2}\right)^{2}\right]\right\}$

$\sigma(\vec{x})=\frac{6 P L}{x_{4} x_{3}^{2}}, \delta(\vec{x})=\frac{6 P L^{3}}{E x_{2}^{2} x_{4}}$

$P_{c}(\vec{x})=\frac{4.013 E \frac{\sqrt{x_{3}^{2} x_{4}^{6}}}{36}}{L^{2}}\left(1-\frac{x_{3}}{2 L} \sqrt{\frac{E}{4 G}}\right)$

\subsection{Cantilever Beam Design Problem}

The fifth optimization problem of civil engineering has been taken into consideration, which is a cantilever beam consisting of five hollow elements with square-shaped cross-section (Fig.7). The objective of this optimization problem is to reduce the weight of the beam. In this design problem, each element is defined by one variable and overall structure consists of 5 structural parameters. The thickness of the beam is taken as constant. In the proposed design, the vertical load is applied to the free end of the beam (node 6) and the right side of the beam (node 1) is rigidly supported. While designing Cantilever Beam Design Problem, the vertical displacement constraint must be taken into consideration and it should not be violated in the final optimal design. The mathematical formulation of the aforementioned design problem has been illustrated below:

The problem formulation is as follows:

Consider

$\vec{x}=\left[x_{1} x_{2} x_{3} x_{4} x_{5}\right]$

Minimize

$f(\vec{x})=0.6224\left(x_{1}+x_{2}+x_{3}+x_{4}+x_{5}\right)$

Subject to

$g(\vec{x})=\frac{61}{x_{1}^{3}}+\frac{37}{x_{2}^{3}}+\frac{19}{x_{3}^{3}}+\frac{7}{x_{4}^{3}}+\frac{1}{x_{5}^{3}} \leq 1$

Variable range $0.01 \leq x_{1}, x_{2}, x_{3}, x_{4}, x_{5} \leq 100$
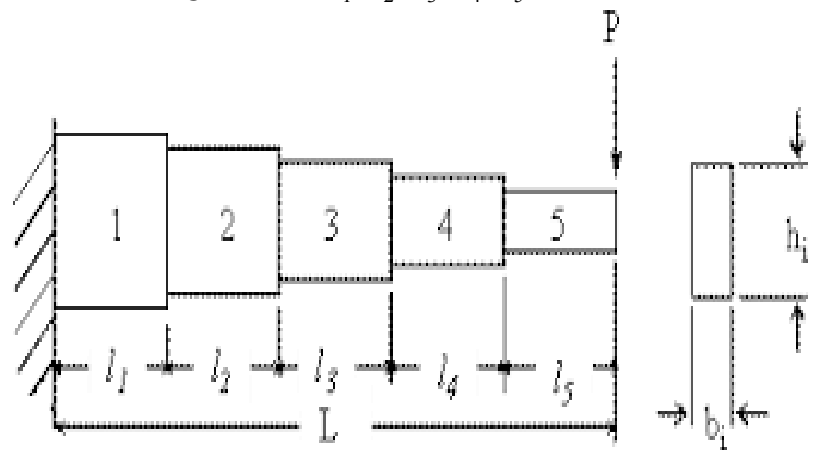

CROSS

SECTION

Fig 7: Cantilever Beam Design Problem

\subsection{Gear Train design Problem}

Gear train design problem is a discrete design problem with four parameters (Fig.8). The major objective this design problem is minimization of the gear ratio, which can be achieved by determining the optimal number of tooth for four gears of a train. In mathematics, the gear train design problem can be described as per the following mathematical equations:

Consider $\vec{x}=\left[x_{1} x_{2} x_{3} x_{4}\right]=\left[N_{A} N_{B} N_{C} N_{D}\right]$

Minimize $f(\vec{x})=\left(\frac{1}{6.931}-\frac{x_{3} x_{4}}{x_{1} x_{4}}\right)^{2}$

Subject to $12 \leq x_{1}, x_{2}, x_{3}, x_{4} \leq 60$ 


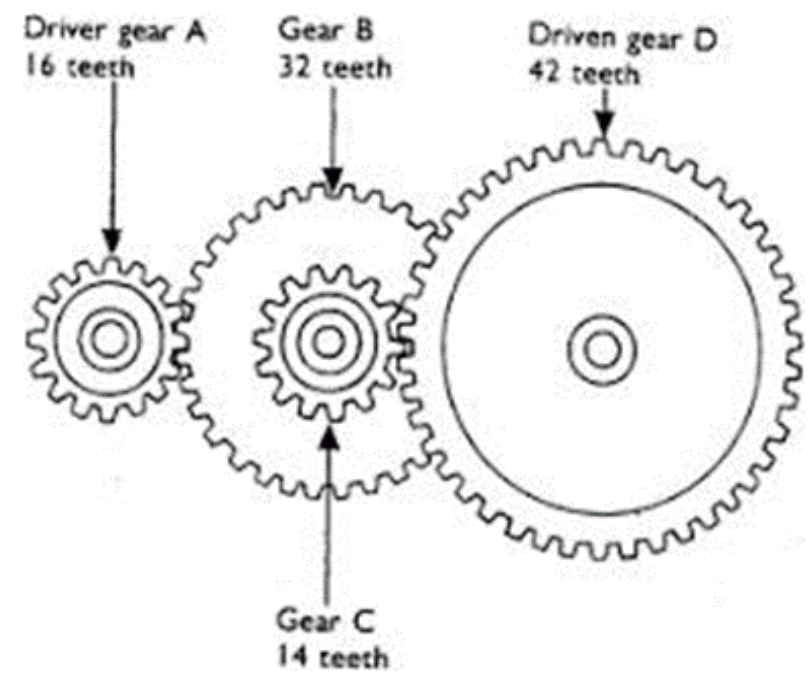

Fig.8: Gear Train design Problem

Table-1 : Solution of Engineering design problems using hGWO-PS

\begin{tabular}{|c|c|c|c|c|c|c|}
\hline $\begin{array}{l}\text { Engineeri } \\
\text { ng Design } \\
\text { Problem }\end{array}$ & $\begin{array}{l}\text { Best } \\
\text { value }\end{array}$ & $\begin{array}{l}\text { Mean } \\
\text { value }\end{array}$ & $\begin{array}{l}\text { Worst } \\
\text { value }\end{array}$ & SD & $\begin{array}{c}\text { med } \\
\text { ian }\end{array}$ & $\begin{array}{c}\text { Wilcoxo } \\
\text { n p- } \\
\text { value }\end{array}$ \\
\hline $\begin{array}{c}\text { Three-bar } \\
\text { truss } \\
\text { problem }\end{array}$ & $\begin{array}{l}263.8 \\
96\end{array}$ & $\begin{array}{l}263.9 \\
01\end{array}$ & $\begin{array}{l}263.91 \\
4\end{array}$ & $\begin{array}{l}0.00 \\
44\end{array}$ & $\begin{array}{l}263 . \\
900\end{array}$ & $1.73 \mathrm{e}-06$ \\
\hline $\begin{array}{c}\text { Pressure } \\
\text { vessel } \\
\text { problem }\end{array}$ & $\begin{array}{l}6160 . \\
106\end{array}$ & $\begin{array}{l}7001 . \\
187\end{array}$ & $\begin{array}{l}7320.3 \\
43\end{array}$ & $\begin{array}{l}391 . \\
305\end{array}$ & $\begin{array}{l}7236 \\
.930\end{array}$ & $1.73 \mathrm{e}-06$ \\
\hline $\begin{array}{c}\text { Tension/c } \\
\text { ompressio } \\
\text { n spring } \\
\text { design } \\
\text { problem }\end{array}$ & $\begin{array}{l}0.012 \\
67\end{array}$ & $\begin{array}{l}0.013 \\
85\end{array}$ & $\begin{array}{l}0.0181 \\
5\end{array}$ & $\begin{array}{l}0.00 \\
10\end{array}$ & $\begin{array}{l}0.01 \\
363\end{array}$ & $73 \mathrm{e}-06$ \\
\hline $\begin{array}{l}\text { Welded } \\
\text { beam } \\
\text { problem }\end{array}$ & $\begin{array}{l}1.726 \\
08\end{array}$ & $\begin{array}{l}1.773 \\
33\end{array}$ & $\begin{array}{l}2.4484 \\
8\end{array}$ & $\begin{array}{l}0.12 \\
8052\end{array}$ & $\begin{array}{l}1.74 \\
77\end{array}$ & $1.73 \mathrm{e}-06$ \\
\hline $\begin{array}{c}\text { Cantilever } \\
\text { Beam } \\
\text { Design }\end{array}$ & $\begin{array}{l}1.303 \\
63\end{array}$ & $\begin{array}{l}1.308 \\
26\end{array}$ & $\begin{array}{l}1.3305 \\
2\end{array}$ & $\begin{array}{l}0.00 \\
51\end{array}$ & $\begin{array}{l}1.30 \\
6\end{array}$ & $1.73 \mathrm{e}-06$ \\
\hline
\end{tabular}

Table-2 : Comparison analysis of Gear Train Design Problem

\begin{tabular}{|c|c|c|c|c|c|}
\hline Algorithm & $\mathbf{N}_{\mathbf{A}}$ & $\mathbf{N}_{\mathbf{B}}$ & $\mathbf{N}_{\mathbf{C}}$ & $\mathbf{N}_{\mathbf{D}}$ & $\begin{array}{c}\text { Optimal Fitness } \\
\text { Value }\end{array}$ \\
\hline MBA [27] & 43 & 16 & 19 & 49 & $2.7009 \mathrm{e}-012$ \\
\hline ISA [28] & --- & --- & --- & --- & $2.7009 \mathrm{e}-012$ \\
\hline GA [29] & --- & --- & --- & --- & $2.33 \mathrm{e}-07$ \\
\hline ABC [30] & 19 & 16 & 44 & 49 & $2.78 \mathrm{e}-11$ \\
\hline GA [31] & 33 & 14 & 17 & 50 & $1.362 \mathrm{e}-09$ \\
\hline ALM [32] & 33 & 15 & 13 & 41 & $2.1469 \mathrm{e}-08$ \\
\hline ALO & 49 & 19 & 16 & 43 & $2.7009 \mathrm{e}-012$ \\
\hline CS [26] & 43 & 16 & 19 & 49 & $2.7009 \mathrm{e}-012$ \\
\hline hGWO-PS & 49 & 19 & 16 & 43 & $2.7009 \mathrm{e}-012$ \\
\hline
\end{tabular}
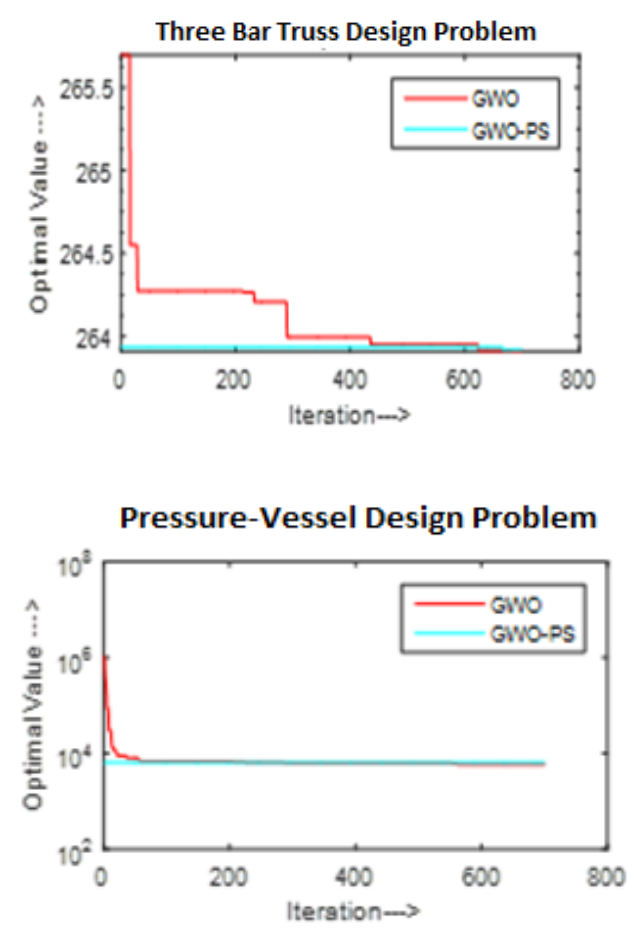

Tension/Compression Spring Design

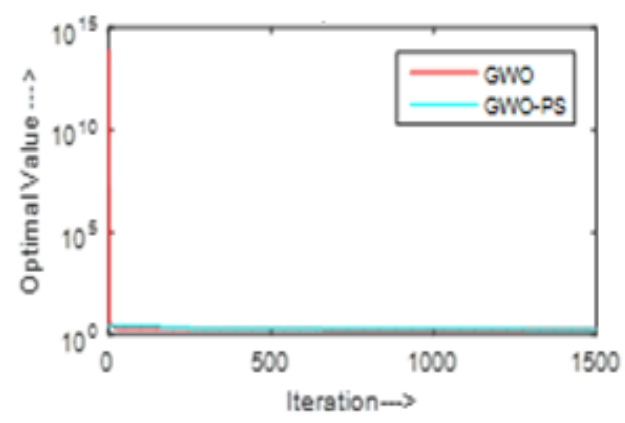

Fig.9: Convergence of GWO and hGWO-PS for various Engineering design problems
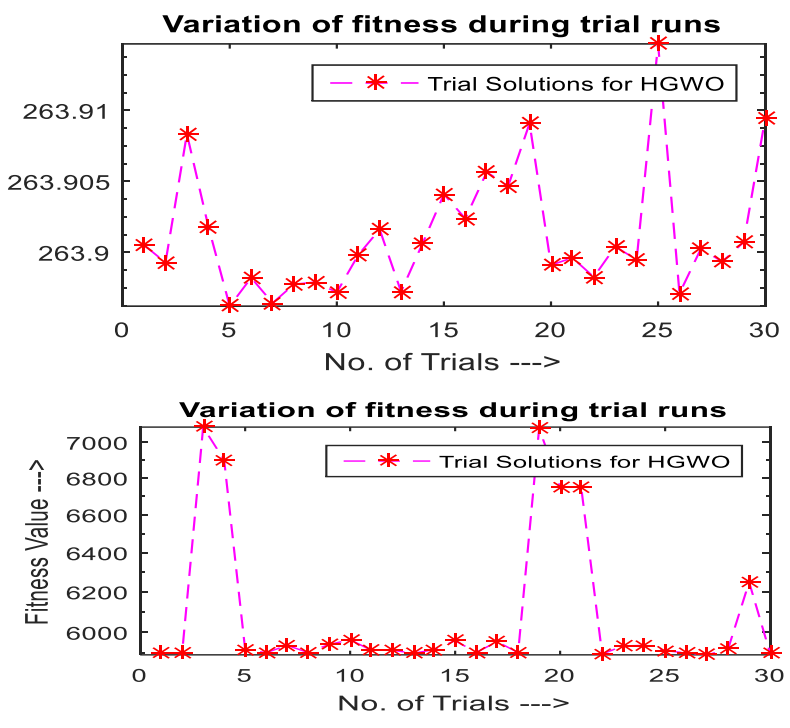

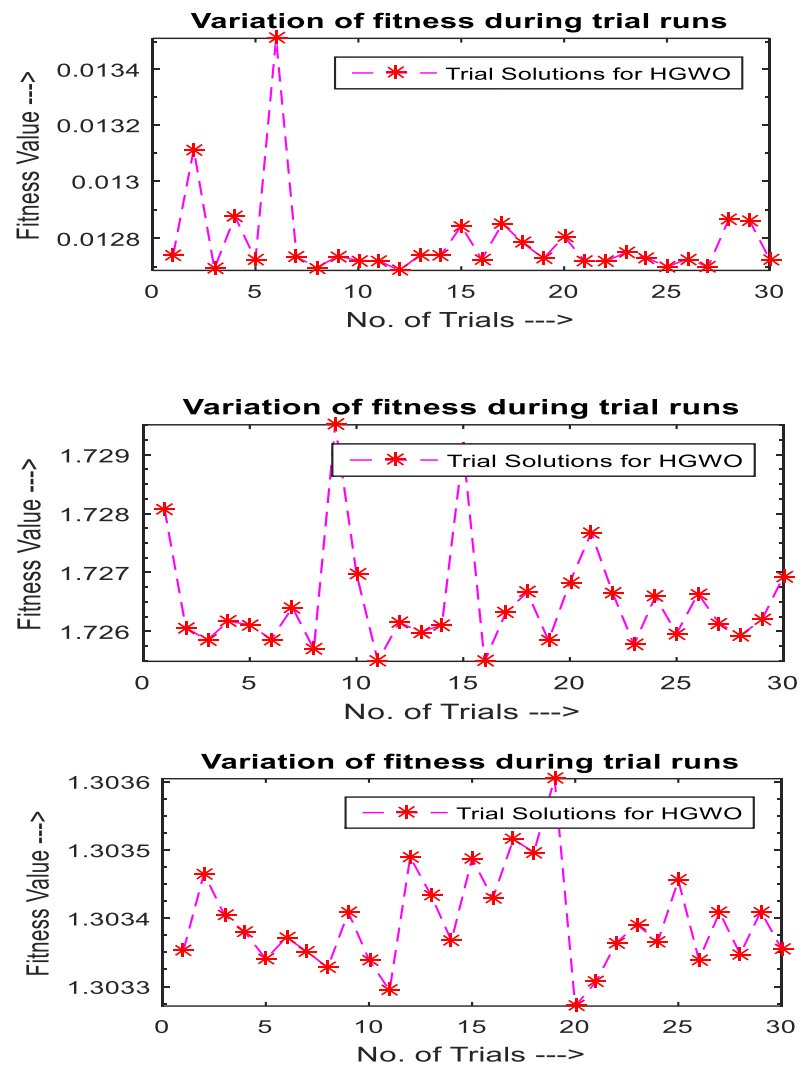

Fig.10: Trial runs solutions of GWO and hGWO-PS for various engineering optimization problems

\section{CONCLUSION}

In the proposed research, the exploitation phase of the existing grey wolf optimizer has been successfully improved using pattern search algorithm and the newly developed hybrid hGWO-PS has been tested for nonlinear, highly constrained, non-convex engineering design and optimization problems. Experimentally, it has been found that the results of the proposed hybrid GWO-PS algorithm are better than existing Grey Wolf Optimizer algorithm, Ant Lion Optimizer algorithm, Moth Flame Optimization algorithm, sine-cosine optimization algorithm and others recently reported heuristics and meta-heuristics search algorithm and hence proposed algorithm indorses its effectiveness in the field of nature inspired meta heuristics algorithms for engineering optimization problems and for improved speed electric vehicle designing.

\section{REFERENCES}

1. D. Simon, "Biogeography-Based Optimization," IEEE Trans. Evol. Comput., vol. 12, no. 6, pp. 702 713, Dec. 2008.

2. S. Mirjalili, S. M. Mirjalili, and A. Lewis, "Grey Wolf Optimizer," Adv Eng Softw, vol. 69, p. 46, 2014.

3. S. Mirjalili, "The ant lion optimizer," $A d v$. Eng. Softw., vol. 83, pp. 80-98, 2015.
4. S. Mirjalili, "Moth-flame optimization algorithm: A novel nature-inspired heuristic paradigm," Knowledge-Based Syst., vol. 89, pp. 228-249, 2015.

5. S. Mirjalili, S. M. Mirjalili, and A. Hatamlou, "Multi-Verse Optimizer: a nature-inspired algorithm for global optimization," Neural Comput. Appl., vol. 27, no. 2, pp. 495-513, 2016.

6. S. Mirjalili, "Dragonfly algorithm: a new metaheuristic optimization technique for solving singleobjective, discrete, and multi-objective problems," Neural Comput. Appl., vol. 27, no. 4, pp. 1053 1073, 2016.

7. S. Mirjalili, "SCA: A Sine Cosine Algorithm for solving optimization problems," Knowledge-Based Syst., vol. 96, pp. 120-133, 2016.

8. H. Shareef, A. A. Ibrahim, and A. H. Mutlag, "Lightning search algorithm," Appl. Soft Comput. $J .$, vol. 36, pp. 315-333, 2015.

9. D. Chaohua, C. Weirong, and Z. Yunfang, "Seeker optimization algorithm," 2006 Int. Conf. Comput. Intell. Secur. ICCIAS 2006, vol. 1, pp. 225-229, 2007.

10. M. D. Li, H. Zhao, X. W. Weng, and T. Han, "A novel nature-inspired algorithm for optimization: Virus colony search," Adv. Eng. Softw., vol. 92, pp. 65-88, 2016.

11. S. Mirjalili and A. Lewis, "The Whale Optimization Algorithm," Adv. Eng. Softw., vol. 95, pp. 51-67, 2016.

12. Z. Bayraktar, M. Komurcu, and D. H. Werner, "Wind Driven Optimization (WDO): A novel nature-inspired optimization algorithm and its application to electromagnetics," 2010 IEEE Int. Symp. Antennas Propag. CNC-USNC/URSI Radio Sci. Meet. - Lead. Wave, AP-S/URSI 2010, no. 1, pp. $0-3,2010$.

13. S. Mirjalili, S. M. Mirjalili, and A. Lewis, "Grey Wolf Optimizer," Adv. Eng. Softw., vol. 69, pp. 4661, 2014.

14. J. G. Vlachogiannis and K. Y. Lee, "Economic load dispatch - A comparative study on heuristic optimization techniques with an improved coordinated aggregation-based PSO," IEEE Trans. Power Syst., vol. 24, no. 2, pp. 991-1001, 2009.

15. M. Herwan Sulaiman, W. Lo Ing, Z. Mustaffa, and M. Rusllim Mohamed, "GREY WOLF OPTIMIZER FOR SOLVING ECONOMIC DISPATCH PROBLEM WITH VALVELOADING EFFECTS,” vol. 10, no. 21, 2015.

16. A. Bhardwaj, V. K. Kamboj, V. K. Shukla, B. Singh, and P. Khurana, "Unit commitment in electrical power system - A literature review," in 2012 IEEE International Power Engineering and Optimization Conference, PEOCO 2012 Conference Proceedings, 2012.

17. S. Mirjalili, "How effective is the Grey Wolf optimizer in training multi-layer perceptrons," Appl. Intell., vol. 43, no. 1, pp. 150-161, Jul. 2015.

18. T.-S. Pan, T.-K. Dao, T.-T. Nguyen, and S.-C. Chu, 
"A Communication Strategy for Paralleling Grey Wolf Optimizer," Springer, Cham, 2016, pp. 253262.

19. M. A. Tawhid and A. F. Ali, "A Hybrid grey wolf optimizer and genetic algorithm for minimizing potential energy function," Memetic Comput., vol. 9, no. 4, pp. 347-359, Dec. 2017.

20. D. Jitkongchuen, "A hybrid differential evolution with grey wolf optimizer for continuous global optimization," in 2015 7th International Conference on Information Technology and Electrical Engineering (ICITEE), 2015, pp. 51-54.

21. S. Zhang, Q. Luo, and Y. Zhou, "Hybrid Grey Wolf Optimizer Using Elite Opposition-Based Learning Strategy and Simplex Method," Int. J. Comput. Intell. Appl., vol. 16, no. 2, p. 1750012, Jun. 2017.

22. N. Mittal, U. Singh, and B. S. Sohi, "Modified Grey Wolf Optimizer for Global Engineering Optimization," Appl. Comput. Intell. Soft Comput., vol. 2016, pp. 1-16, May 2016.

23. N. Singh and S. B. Singh, "A Modified Mean Gray Wolf Optimization Approach for Benchmark and Biomedical Problems.," Evol. Bioinform. Online, vol. 13, p. 1176934317729413, 2017.

24. N. Singh and S. B. Singh, "Hybrid Algorithm of Particle Swarm Optimization and Grey Wolf Optimizer for Improving Convergence Performance," J. Appl. Math., vol. 2017, pp. 1-15, Nov. 2017.

25. S. Gupta and K. Deep, "A novel Random Walk Grey Wolf Optimizer," Swarm Evol. Comput., Jan. 2018.

26. A. H. Gandomi, Y. X-s, and A. A. C. search algorithm:, "a metaheuristic approach to solve structural optimization problems," Eng Comput, vol. 29, no. 17, p. 35, 2013.

27. A. Sadollah, A. Bahreininejad, H. Eskandar, and H. M. M. blast algorithm:, "a new population based algorithm for solving constrained engineering optimization problems," Appl Soft Comput, vol. 13, no. 2592 , p. 612,2013

28. G. Ah., Interior search algorithm (ISA): a novel approach for global optimization. ISA Trans, 2014.

29. W. S-j and C. P-t., "Genetic algorithms for nonlinear mixed discrete-integer optimization problems via meta-genetic parameter optimization," Eng Optim + A, vol. 3524, no. 137, p. 59, 1995.

30. T. K. Sharma, M. Pant, and V. Singh, "Improved local search in artificial bee colony using golden section search. arXiv," 2012.

31. K. Deb and M. A. Goyal, "combined genetic adaptive search (GeneAS) for engineering design," Comput Sci Inf., vol. 26, no. 30, p. 45, 1996.

32. B. Kannan and K. Sn., No Title. An augmented Lagrange multiplier based method for mixed integer discrete continuous optimization.

33. Seyedali Mirjalili,The Ant Lion Optimizer, Advances in Engineering Software, Volume 83,2015, Pages 80-98, ISSN 0965-9978, https://doi.org/10.1016/j.advengsoft.2015.01.010. 